\title{
Leaders
}

\section{Gynaecological effects of tamoxifen}

\author{
Sezgin M Ismail
}

"He's the best physician that knows the worthlessness of the most medicines"

From Poor Richard's Almanack, Benjamin Franklin, 1733

The non-steroidal triphenylethylene derivative tamoxifen has been widely used in the management of established breast cancer for some 25 years. It prolongs overall survival and diseasefree survival in women with breast cancer. ${ }^{1-3}$ It also reduces the risk of contralateral disease in treated women with breast cancer. ${ }^{2}{ }^{5}$ This last observation resulted in large scale, randomised, placebo controlled studies in the United Kingdom, Italy, and the USA, designed to assess the efficacy of tamoxifen in asymptomatic women at increased risk of breast cancer by virtue of a strong family history and other predisposing factors. The American prevention study has recently been discontinued earlier than planned, having allegedly demonstrated a $45 \%$ reduction in breast cancer among tamoxifen treated women. ${ }^{67}$ The preliminary results from the Italian and Royal Marsden studies have been unable to confirm a substantial reduction in breast cancer in the tamoxifen treated women. ${ }^{89}$ Nevertheless, in the wake of the publicity generated by the findings of the American study, tamoxifen consumption looks set to increase for the foreseeable future, not only among women with breast cancer but also among well women with a family history of breast cancer. Since the optimal duration of tamoxifen treatment has not yet been fully established in either context, ${ }^{10}$ it is likely that treatment will continue for long periods in both groups of women. I would predict, therefore, that side effects of tamoxifen will be seen with increasing frequency.

Although tamoxifen is generally well tolerated and relatively non-toxic, it has become clear during the past decade that prolonged use of this agent is associated with significant gynaecological complications. These include functional ovarian cysts in pre- and perimenopausal women and proliferative endometrial abnormalities in postmenopausal women. In this article, I will review critically the literature on the gynaecological effects of prolonged tamoxifen treatment and consider the role of tamoxifen in endometrial carcinogenesis.

\section{Cervical effects of tamoxifen}

The first indication that tamoxifen, "an antioestrogen," might have complex effects on the human female genital tract came in 1977 with the observation that tamoxifen treated postmenopausal women had oestrogenised rather than atrophic smears. ${ }^{11}$ Since the oestrogenic changes regressed after cessation of tamoxifen treatment, the authors concluded that they were caused by the drug. The oestrogenic effects of tamoxifen on cervicovaginal squamous epithelial cells of postmenopausal women has since been repeatedly confirmed. ${ }^{12-14}$ Some $89 \%$ of tamoxifen treated postmenopausal breast cancer patients develop oestrogenised cervical smears despite serum oestradiol levels within the postmenopausal range. ${ }^{14}$

\section{Endometrial effects of tamoxifen}

Because breast cancer is predominantly a disease of older women, published reports on the endometrial effects of tamoxifen pertain largely to postmenopausal endometria. Limited information is available about the effects of tamoxifen on the endometrium of premenopausal women. McGonigle et al confirmed a high incidence of endometrial polyps among postmenopausal tamoxifen treated women, but failed to show a statistically significant increase in polyps among the premenopausal subjects. ${ }^{15}$ In a more recent study there was a high prevalence of pathological findings in the endometrium of symptomatic tamoxifen treated women, but this finding was far more marked in postmenopausal than in premenopausal women. ${ }^{16}$ Thus it is not clear whether tamoxifen has the same effects on the premenopausal endometrium as on the postmenopausal endometrium. The prevention trials should yield more information about the effects of tamoxifen on premenopausal endometria, but the ensuing discussion refers largely to postmenopausal women.

Published reports on the pathological effects of tamoxifen on the endometrium are difficult to evaluate critically because of an unfortunate lack of attention to pathological detail and a lack of understanding of the pathological terminology. In addition, there is a tendency to make extrapolations from ultrasonographic and hysteroscopic findings without a detailed tissue diagnosis. In many cases, a tissue diagnosis cannot be established because tamoxifen treated endometrium is notoriously difficult to biopsy even in the presence of ultrasonographic or hysteroscopic abnormalities. In one study, an endometrial biopsy was attempted in 77 
tamoxifen treated postmenopausal breast cancer patients, but adequate endometrial tissue was obtained in only 22 cases (29\%), even though 76 women had an ultrasonographically thickened endometrium. ${ }^{17}$ Nevertheless, it appears that tamoxifen treated postmenopausal breast cancer patients have a significantly higher incidence of proliferative endometrial abnormalities (endometrial hyperplasia, polyps, and malignant neoplasms) than untreated postmenopausal women with breast cancer.

ENDOMETRIAL HYPERPLASIA

Normal postmenopausal endometrium is atrophic. By definition, the term atrophy denotes lack of growth and a diminution in the amount of the affected tissue. Thus normal postmenopausal endometrium is thin and shows no mitotic activity.

Various investigators have noted that the endometrium of tamoxifen treated postmenopausal breast cancer patients is significantly thicker on ultrasonographic examination than that of untreated controls. ${ }^{18-20}$ More recently, Lindahl et al have shown that breast cancer patients treated with tamoxifen develop a statistically significant increase in ultrasonographic endometrial thickness, while no such change was observed in patients treated in other ways. ${ }^{21}$

Because of the difficulties of sampling tamoxifen stimulated endometrium, limited information is available about its pathological features. However, the ultrasonographic observations documenting tamoxifen stimulated endometrial thickening are in keeping with reports of an increased incidence of proliferative activity/ hyperplasia in the endometrium of tamoxifen treated breast cancer patients. ${ }^{17}{ }^{20}{ }^{22-24}$ Neven et al found proliferative/hyperplastic features in the endometrium of $43 \%$ of tamoxifen treated breast cancer patients compared with $14 \%$ of untreated patients. ${ }^{22}$ Cohen et al found proliferative activity in 19 of 22 endometrial biopsies obtained from a series of 77 asymptomatic tamoxifen treated postmenopausal breast cancer patients. ${ }^{17}$

On macroscopic examination, tamoxifen stimulated endometrial hyperplasia is characterised by diffuse endometrial thickening with a Swiss-cheese-like cut surface owing to the presence of multiple intraendometrial cysts of varying size. ${ }^{25}$ Microscopic examination confirms that there is diffuse endometrial thickening with cystically dilated glands which are clearly of endometrial origin and located within the endometrium, ${ }^{25}$ rather than beneath it as has been suggested on the basis of ultrasonographic findings. ${ }^{26}$ Some endometrial glands show glandular budding and other architectural abnormalities. Focally, the glandular epithelium shows mitotic activity and mucinous, clear cell, oxyphilic, and other epithelial metaplasias are often seen. The endometrial stroma is characteristically fibrotic ${ }^{2527}{ }^{28}$ with collagen bundles separating stromal cells. $^{27}$ This feature of tamoxifen stimulated endometrium may be responsible for the difficulty in obtaining endometrial biopsy material from treated women with documented ultrasonographic and hysteroscopic abnormalities. Occasional stromal mitoses may also be seen. These microscopic appearances, which indicate an increase in the amount of endometrial tissue and a low but definite proliferative activity, are in my opinion best interpreted as hyperplasia of the endometrium.

Recently, there have been suggestions that the ultrasonographically detectable thickened cystic endometrium which many tamoxifen treated women develop represents cystic atrophy rather than hyperplasia. ${ }^{28-30}$ The only investigators with this view who present pathological findings in some detail document the presence of proliferative activity in the lesion they term cystic atrophy. ${ }^{30}$ Thus the suggestion that tamoxifen stimulated endometrial growth represents atrophy would seem to be a pathological oxymoron.

\section{ENDOMETRIAL POLYPS}

Prolonged tamoxifen treatment is associated with a significant increase in the incidence of endometrial polyps. ${ }^{151618202231}$ Neven et al reported a sevenfold increase in endometrial polyps among tamoxifen treated asymptomatic breast cancer patients when compared with patients not treated with tamoxifen. ${ }^{22}$ A larger study by Lahti et al found endometrial polyps in $36 \%$ of treated and $10 \%$ of untreated asymptomatic postmenopausal breast cancer patients. ${ }^{18}$

Endometrial polyps which develop in tamoxifen treated women have unusual macroscopic and microscopic features. ${ }^{25} 3233$ Tamoxifen associated endometrial polyps tend to develop on a background of endometrial hyperplasia. ${ }^{25}$ The polyps are often multiple and usually much larger than sporadic polyps. ${ }^{25}{ }^{32}$ They may undergo ulceration and infarction and the consequent irregular friable appearance may mimic malignancy. Gross myxoid change is occasionally seen.

On microscopic examination, the polyps comprise abundant fibromyxoid stroma and architecturally abnormal endometrial glands. Proliferative activity is commonly seen in both epithelial and stromal cells. The epithelial cells lining the glands show a range of metaplasias including apocrine, squamoid, mucinous, clear cell, and oxyphil cell metaplasia. There is patchy condensation of the stromal cells around the glands. Some investigators have reported stromal decidualisation in tamoxifen polyps, ${ }^{33}$ but in my experience this finding is confined to patients who have received progestogens for their gynaecological symptoms.

The combination of periglandular stromal condensation and mitotic activity seen in tamoxifen associated endometrial polyps may raise the suggestion of müllerian adenosarcoma. The latter is characterised by a frankly sarcomatous stroma with generalised hypercellularity, widespread periglandular stromal condensation, marked cytonuclear atypia, and a relatively high mitotic rate. The reported stromal mitotic rate for müllerian adenosarcomas has ranged from one to 40 mitotic figures per 10 high power fields. ${ }^{34}$ By contrast, tamoxifen 
associated endometrial polyps have a relatively hypocellular stroma, patchy rather than generalised periglandular condensation, and little or no cytonuclear atypia. The stromal mitotic rate in tamoxifen polyps is relatively low, although precise figures are unavailable to date. ${ }^{27}$

There is a high prevalence of endometrial carcinoma arising from tamoxifen associated endometrial polyps, that is, polyp-cancers. Two such cases were noted in a series of seven women with tamoxifen associated endometrial polyps. ${ }^{32}$ Another study of 19 tamoxifen treated women documented three instances of this phenomenon $^{25}$ which is extremely uncommon outside a tamoxifen setting. Precise figures are not available for the general population, but only four polyp-cancers were found in a large study of 1100 endometrial polyps carried out before the introduction of tamoxifen. ${ }^{35}$

ENDOMETRIAL NEOPLASMS

The pioneering study of Fornander et al was the first to show a statistically significant increase in endometrial cancer among tamoxifen treated postmenopausal breast cancer patients. ${ }^{4}$ This study included 1846 postmenopausal women with early breast cancer, of whom 931 were randomised to receive tamoxifen $40 \mathrm{mg} /$ day. There were 13 invasive endometrial carcinomas among the tamoxifen group over a median follow up of 4.5 years. There were two cases of corpus cancer among the placebo group: a uterine leiomyosarcoma and a malignant mixed müllerian tumour. The risk ratio for corpus cancer was 6.4 (95\% confidence interval 1.4 to 28 )

Although heavily criticised when first published, this classic study has withstood the test of time. Other large well designed studies have confirmed that women with breast cancer who are treated with tamoxifen are at increased risk of endometrial carcinoma when compared with breast cancer patients who are not treated with this drug. ${ }^{36-38} \mathrm{~A}$ recent overview of the randomised tamoxifen trials found that the incidence of endometrial cancer was approximately doubled in trials of tamoxifen lasting one or two years, and approximately quadrupled in trials of five years' duration. ${ }^{3}$ The discontinued American breast cancer prevention trial, in which 13388 women were randomised to receive placebo or tamoxifen 20 mg a day, found 33 cases of endometrial cancer among tamoxifen treated women and 14 cases in the placebo group. ${ }^{6}$ Thus the causal link between prolonged tamoxifen treatment and endometrial carcinoma is now established beyond all reasonable doubt.

There is, however, still some uncertainty about the pathological features of tamoxifen associated endometrial cancer. In a small retrospective study, ${ }^{39}$ it was found that $67 \%$ of 15 tamoxifen treated breast cancer patients who developed endometrial carcinoma had high grade endometrioid adenocarcinomas or histological subtypes associated with a poor prognosis. Only nine of the 38 untreated women with endometrial cancer had poor prognosis tumours. Five of the tamoxifen treated women and one of the untreated women died of endometrial cancer.

In an even smaller study, ${ }^{40}$ six cases of endometrial carcinosarcoma were documented in tamoxifen treated women, suggesting a causal link between prolonged tamoxifen treatment and this subtype of endometrial carcinoma which is associated with a poor prognosis.

In another small study, ${ }^{31}$ it was found that serous type of endometrial carcinoma, which has a particularly poor prognosis, was the commonest subtype of endometrial carcinoma among tamoxifen treated breast cancer patients $(n=15)$, while endometrioid carcinoma was the commonest subtype among those not treated with tamoxifen $(n=57)$. The difference between the tamoxifen and nontamoxifen patients was statistically significant in this respect but, despite the poor prognosis associated with the serous type of endometrial carcinoma, there was no significant difference in tumour stage or clinical outcome between the two groups.

On the other hand, a large case-control study ${ }^{37}$ found no significant difference in stage, morphology, and death from endometrial cancer in 98 women with tamoxifen associated endometrial cancer and 285 non-tamoxifentreated breast cancer patients who subsequently developed endometrial cancer. In contrast to the reports above, this study showed an excess of well differentiated tumours in tamoxifen users (52\%) compared with nonusers $(32 \%)$. Other sizeable studies ${ }^{41}$ have also been unable to show a specific association between tamoxifen and poorly differentiated or aggressive endometrial carcinomas.

\section{OTHER UTERINE PATHOLOGY}

Cohen et al found that eight of 14 hysterectomy specimens from tamoxifen treated postmenopausal breast cancer patients contained adenomyosis. ${ }^{43}$ Since adenomyosis is otherwise relatively rare in postmenopausal women, these investigators postulated a causal relation between tamoxifen and adenomyosis in these patients.

A few cases of endometrial stromal sarcoma have been reported in association with tamoxifen treatment, ${ }^{44}$ and Clement et al have documented a series of six müllerian adenosarcomas which developed in tamoxifen treated women. ${ }^{46}$

\section{Ovarian effects}

Ovarian cysts have also been reported in association with prolonged tamoxifen use. $.^{20} 47-49$ These have been found to occur in both premenopausal $^{47} 48$ and postmenopausal ${ }^{2049}$ women receiving tamoxifen. In premenopausal women the cysts may be associated with hyperoestrogenism. ${ }^{47} 48$ The pathology of these cysts is poorly documented but those occurring in premenopausal women have been variously reported as luteinised or follicular cysts, ${ }^{49}$ benign unilateral corpus luteum cysts, ${ }^{48}$ or bilateral "simple" functional cysts" ${ }^{47}$ which were presumably luteinised follicle cysts. The 
ovarian cysts which develop in tamoxifen treated postmenopausal women may represent cystic ovarian tumours ${ }^{50}$ or endometriotic cysts. ${ }^{51-53}$

Cohen et al reported that 10 of 16 tamoxifen treated women who underwent total abdominal hysterectomy and bilateral salpingooophorectomy had ovarian neoplasms. ${ }^{50}$ Four of these women had bilateral ovarian serous cystadenomas, one had bilateral serous cystadenofibromas, and another had a unilateral serous cystadenofibroma. Other ovarian tumours which occurred in this group included an endometrioid adenocarcinoma, a Brenner tumour, a thecoma, and ovarian fibromas. It is important to emphasise, however, that women with breast cancer, particularly those with a family history, may already have a predisposition to ovarian neoplasia and that this small study by no means establishes a link between tamoxifen and ovarian tumorigenesis.

\section{Endometriosis}

English language reports to date contain eight cases of tamoxifen associated endometriosis, of which five have been in premenopausal ${ }^{54-57}$ and three in postmenopausal women. ${ }^{51-53}$ Endometriosis is common in premenopausal women and its occurrence in tamoxifen treated women in this age group may therefore be coincidental. In contrast, endometriosis is rare in postmenopausal women and the finding of endometriosis in postmenopausal women treated with tamoxifen raises the possibility of a link between tamoxifen and endometriosis. This possibility is further supported by the unusual morphological features and behaviour of endometriosis in tamoxifen treated postmenopausal women..$^{51-53}$ One patient had pelvic endometriosis infiltrating the cervix, vagina, rectum, and sigmoid colon. ${ }^{51}$ Another patient had a cystic ovarian mass with mucinous contents, friable masses on its serosal surface, and apparent infiltration of adjacent structures. ${ }^{52}$ On pathological examination, this was shown to be an endometriotic cyst with features identical to those described in tamoxifen treated endometrium including epithelial metaplasias and polyp formation. Another patient developed an ovarian endometrioid carcinoma in an endometriotic cyst, ${ }^{53}$ suggesting that prolonged tamoxifen treatment may be associated with an increased risk of carcinoma arising in endometriotic foci.

\section{Mechanisms of action on the female genital tract}

The mechanisms by which tamoxifen effects these changes in the female genital tract are poorly understood. Some effects may be mediated through the oestrogen receptor, but it has become clear during the past decade that tamoxifen also has other, oestrogen receptor independent effects. The drug may affect intracellular signal transduction by acting directly on cell membranes. ${ }^{58-63}$ It has oestrogen receptor independent effects on apoptosis ${ }^{64} 65$ and local growth control mechanisms. ${ }^{66-75}$ In premenopausal women, it may disrupt the function of the hypothalamic-pituitaryovarian axis. $^{76-80}$

\section{Tamoxifen and endometrial} carcinogenesis

Tamoxifen is carcinogenic in laboratory rodents, in which it induces liver tumours, acting both as initiator and promoter. ${ }^{81-84}$ Recently it has been reported that mice exposed to tamoxifen in the neonatal period often develop endometrial hyperplasia and adenocarcinoma. ${ }^{85}$ Tamoxifen administration produces DNA adducts in the liver of rats, hamsters, and mice, the concentration of which is related to the dose and duration of tamoxifen treatment. ${ }^{86-88}$ However, to date no tamoxifen induced DNA adducts have been detected in the uterus of experimental animals. It is not clear if tamoxifen has genotoxic effects on human endometrium as evidence to date is contradictory, ${ }^{89}{ }^{90}$ but its potential growth promoting effect is well established.

Tamoxifen induced endometrial abnormalities form a pathological spectrum which comprises endometrial hyperplasia, endometrial polyps arising on a background of hyperplasia, adenocarcinomas arising in polyps, and overtly invasive adenocarcinomas. ${ }^{25} \mathrm{It}$ is likely therefore that polyps represent an important intermediate stage in this pathway of endometrial carcinogenesis. The factors which predispose to the development of endometrial hyperplasia in tamoxifen treated women and to progression through this pathological spectrum remain to be determined.

1 Early Breast Cancer Triallists' Collaborative Group. Effects of adjuvant tamoxifen and of cytotoxic therapy on mortality in early breast cancer: an overview of 61 randomized trials among 28,896 women. $N$ Engl f Med 1988;319:1681-92.

2 Early Breast Cancer Triallists' Collaborative Group. Systemic treatment of early breast cancer by hormonal, cytotoxic or immune therapy. 133 randomized trials involving 31,000 recurrences and 24,000 deaths among 75,000 women. Lancet 1992;339:1-15.

3 Early Breast Cancer Triallists' Collaborative Group. Tamoxifen for early breast cancer: an overview of the randomised trials. Lancet 1998;351:1451-67.

4 Fornander T, Rutqvist LE, Cedermark B, et al. Adjuvant tamoxifen in early breast cancer: occurrence of new primary cancers. Lancet $1989 ; \mathbf{i}: 117-20$.

5 Fisher B, Costantino J, Redmond C, et al. A randomized clinical trial evaluating tamoxifen in the treatment of patients with node negative breast cancer who have estrogen-receptor-positive tumors. N Engl F Med 1989;320: $479-84$

6 Josefson D. Breast cancer trial stopped early. BMF 1998;316:1187.

7 Marshall E. Tamoxifen - "a big deal," but a complex hand to play. Science $1998 ; 280: 196$.

8 Veronesi U, Maisonneuve P, Costa A, et al. Prevention of breast cancer with tamoxifen: preliminary findings from the Italian randomised trial among hysterectomised women. Lancet 1998;352:93-7.

9 Powles T, Eeles R, Ashley S, et al. Interim analysis of the incidence of breast cancer in the Royal Marsden Hospital tamoxifen randomised chemoprevention trial. Lancet 1998; 352:98-101.

10 Rea D, Poole C, Gray R. Adjuvant tamoxifen: how long before we know how long? BMF 1998;316:1518-19.

11 Ferrazzi E, Cartei G, Mattarazzo R, et al. Oestrogen-like effect of tamoxifen on vaginal epithelium. BMF 1977;i: 1351-2.

12 Boccardo F, Bruzzi P, Rubagotti A, et al. Estrogen-like action of tamoxifen on vaginal epithelium of breast cancer patients. Oncology 1981;30:281-5.

13 Eells TP, Alpern HD, Grzywacz C, et al. The effect of tamoxifen on cervical squamous maturation in Papanicolau stained cervical smears of postmenopausal women. Cytopathology 1990;1:263-8.

14 Lahti E, Vuopala S, Kauppila A, et al. Maturation of vaginal and endometrial epithelium in postmenopausal breast cancer patients receiving long-term tamoxifen. Gynecol Oncol 1994;55:410-14.

15 McGonigle KF, Lantry SA, Odommaryon TL, et al. Histopathologic effects of tamoxifen on the uterine epithelium of breast cancer patients - analysis by menopausal status. Cancer Lett 1996;101:59-66. 
16 Cheng WF, Lin HH, Torng PL, et al. Comparison of endometrial changes among symptomatic tamoxifentreated and nontreated premenopausal and postmenopau233-7.

17 Cohen I, Rosen DJD, Shapira J, et al. Endometrial changes in postmenopausal women treated with tamoxifen for breast cancer. Br f Obstet Gynaecol 1993;100:567-70.

18 Lahti E, Guillermo B, Kauppila A, et al. Endometrial changes in postmenopausal breast cancer patients receiving tamoxifen. Obstet Gynecol 1993;81:660-4.

19 Cohen I, Rosen DJD, Shapira J, et al. Endometrial changes with tamoxifen: comparison between tamoxifen-treated and nontreated asymptomatic, postmenopausal breast cancer patients. Gynecol Oncol 1994;52:185-90.

20 Kedar RP, Bourne TH, Powles TJ, et al. Effects of tamoxifen on uterus and ovaries of postmenopausal women in a randomised breast cancer prevention trial. Lancet 1994;343 1318-21.

21 Lindahl B, Andolf E, Ingvar C, et al. Endometrial thickness and ovarian cysts as measured by ultrasound in asymptomatic postmenopausal breast cancer patients on various adjuvant treatments including tamoxifen. Anticancer Res 1997; 17:3821-4.

22 Neven P, De Muylder X, Van Belle Y, et al. Tamoxifen and the uterus and endometrium. Lancet 1989;i:375.

23 Neven P, De Muylder X, Van Belle Y, et al. Hysteroscopic follow-up during tamoxifen treatment. Eur f Obstet Gynecol Reprod Biol 1990;35:235-8.

24 De Muylder X, Neven P, De Somer M, et al. Endometrial lesions in patients undergoing tamoxifen therapy. Int $\mathscr{f}$ Gynecol Oncol 1991;36:127-30.

25 Ismail SM. Pathology of endometrium treated with tamoxifen. F Clin Pathol 1994;47:827-33.

26 Goldstein SR. Unusual ultrasonographic appearance of the uterus in patients receiving tamoxifen. Am f Obstet Gynecol 1994;170:447-51.

27 Ismail SM. Endometrial pathology associated with prolonged tamoxifen therapy. A review. Adv Anat Pathol 1996; 3:266-71.

28 Touraine P, Driguez P, Cartier I, et al. Lack of induction of endometrial hyperplasia with tamoxifen. Lancet 1995;345: $254-5$

29 Neven P, De Muylder X, Van Belle Y, et al. Longitudinal hysteroscopic follow-up during tamoxifen treatment. Lancet 1998;351:36.

30 McGonigle KF, Shaw SL, Vasilev SA, et al. Abnormalities detected on transvaginal ultrasonography in tamoxifentreated postmenopausal breast cancer patients may represent endometrial cystic atrophy. Am f Obstet Gynecol 1998; 178: $1145-50$

31 Silva EG, Tornos CS, Follen-Mitchell M. Malignant neoplasms of the uterine corpus in patients treated for breast carcinoma: the effects of tamoxifen. Int 7 Gynecol Pathol 1994;13:248-58.

32 Nuovo MA, Nuovo GJ, McCaffrey RM, et al. Endometrial polyps in postmenopausal patients receiving tamoxifen. Int polyps in postmenopausal patients

33 Corley D, Rowe J, Curtis MT, et al. Postmenopausal bleeding from unusual endometrial polyps in women on chronic ing from unusual endometrial polyps in women on

34 Clement PB, Scully RE. Mullerian adenosarcoma of the uterus: a clinicopathologic analysis of 100 cases with a review of the literature. Hum Pathol 1990;21:363-81.

35 Peterson WF, Novak ER. Endometrial polyps. Obstet Gynecol $1956 ; 8: 40-9$.

36 Fisher B, Costantino JP, Redmond CK, et al. Endometrial cancer in tamoxifen-treated breast cancer patients: findings from the National Surgical Adjuvant Breast and Bowel Project (NSABP) B-14. F Natl Cancer Inst 1994;86:527-37.

37 Van Leeuwen FE, Benraadt J, Coebergh JWW, et al. Risk of endometrial cancer after tamoxifen treatment of breast endometrial cancer after tamoxife
cancer. Lancet 1994;343:448-52.

38 Rutqvist LE, Johansson H, Signomklao T, et al. Adjuvant tamoxifen therapy for early stage breast cancer and second primary malignancies. $\mathcal{F}$ Natl Cancer Inst 1995;87:645-51.

39 Magriples U, Naftolin F, Schwartz PE, et al. High-grade endometrial carcinoma in tamoxifen-treated breast cancer patients. F Clin Oncol 1993;11:485-90.

40 Evans MJ, Langlois NEI, Kitchener HC, et al. Is there an association between long-term tamoxifen treatment and the development of carcinosarcoma (malignant mixed müllerian tumor) of the uterus. Int $\mathcal{F}$ Gynecol Cancer 1995; 5:310-13.

41 Barakat RR, Wong G, Curtin JP, et al. Tamoxifen use in breast cancer patients who subsequently develop corpus cancer is not associated with a higher incidence of adverse histologic features. Gynecol Oncol 1994;55:164-8.

42 Mignotte H, Rodier JF, Lesur A, et al. Endometrial carcinoma associated with adjuvant therapy for breast cancer: a French multi-centre analysis of 89 cases. Breas $1995 ; 4: 200-2$

43 Cohen I, Beyth Y, Tepper R, et al. Adenomyosis in postmenopausal breast-cancer patients treated with tamoxifen-a new entity. Gynecol Oncol 1995;58:86-91.

44 Beer TW, Buchanan R, Buckley CH. Uterine stromal sarcoma following tamoxifen treatment. F Clin Pathol 1995; 48:596.

45 Eddy GL, Mazur MT. Endolymphatic stromal myosis associated with tamoxifen use. Gynecol Oncol 1997;64:262-4.

46 Clement PB, Oliva E, Young RH. Mullerian adenosarcoma of the uterine corpus associated with tamoxifen therapy. Int f Gynecol Pathol 1996;15:222-9.
47 Cohen I, Rosen DJD, Altaras M, et al. Tamoxifen treatment in premenopausal breast cancer patients may be associated with ovarian overstimulation, cystic form

48 Hochner-Celnikier D, Anteby E, Yagel S. Ovarian cysts in tamoxifen-treated premenopausal women with breast cancer-a management dilemma. Am f Obstet Gynecol 1995;172:1323-4.

49 Shushan A, Peretz T, Uziely B, et al. Ovarian cysts in premenopausal and postmenopausal tamoxifen-treated women with breast cancer. Am f Obstet Gynecol 1996;174: $141-4$.

50 Cohen I, Beyth Y, Tepper R, et al. Ovarian tumors in postmenopausal breast cancer patients treated with tamoxifen. Gynecol Oncol 1996:60:54-8.

51 Hajjar LR, Kim WS, Nolan GH, et al. Intestinal and pelvic endometriosis presenting as a tumor and associated with tamoxifen therapy: report of a case. Obstet Gynecol 1993;82:642-4

52 Ismail SM, Maulik TG. Tamoxifen-associated postmenopausal endometriosis. Histopathology 1997;30:187-91

53 Cohen I, Altaras MM, Lew S, et al. Ovarian endometrioid carcinoma and endometriosis developing in a postmenopausal breast cancer patient during tamoxifen therapy: a case report and review of the literature. Gynecol Oncol 1994;55:443-7.

54 Ford MRW, Turner MJ, Wood C, et al. Endometriosis developing during tamoxifen therapy. Am f Obstet Gynecol 1988;158:1119.

55 Cano A, Metallin P, Legua V, et al. Tamoxifen and the uterus and endometrium. Lancet 1989;i:376.

56 Buckley $\mathrm{CH}$. Tamoxifen and endometriosis. Case report. $\mathrm{Br}$ f Obstet Gynaecol 1990;97:645-6.

57 Morgan MA, Gincherman Y, Mikuta JJ. Endometriosis and tamoxifen therapy. Int $\mathcal{F}$ Gynecol Obstet 1994;45:55-7.

58 Cabot MC, Zhang ZC, Giuliano AE. Tamoxifen elicits rapid transmembrane lipid signal responses in human breast cancer cells. Breast Cancer Res Treat 1995;36:299-306.

59 Charlier C, Bruyneel E, Lechanteur C, et al. Enhancement of tamoxifen-induced E-cadherin function by $\mathrm{Ca}^{2+}$ channel antagonists in human breast cancer MCF7/6 cells. Eur $\mathcal{F}$ Pharmacol 1996;317:413-16.

60 Song JB, Standley PR, Zhang F, et al. Tamoxifen (estrogen antagonist) inhibits voltage-gated calcium current and contractility in vascular smooth muscle from rats. $\mathcal{F}$ Pharmacol Exp Ther 1996;277:1444-53.

61 Gundimeda U, Chen ZH, Gopalakrishna R. Tamoxifen modulates protein-kinase-C via oxidative stress in estrogen receptor-negative breast cancer cells. F Biol Chem 1996; 271:13504-14.

62 Cabot MC, Zhang ZC, Cao HT, et al. Tamoxifen activates cellular phospholipase $\mathrm{C}$ and $\mathrm{D}$ and elicits protein kinase $\mathrm{C}$ translocation. Int f Cancer 1997;70:567-74.

63 Duh JL, Yu R, Jiao JJ, et al. Activation of signal transduction kinases by tamoxifen. Pharm Res 1997;14:186-9.

64 Perry RR, Kang Y, Greaves B. Effects of tamoxifen on growth and apoptosis of estrogen-dependent and estrogenindependent human breast cancer cells. Ann Surg Oncol 1995;2:238-45.

65 Kang Y, Cortina R, Perry RR. Role of c-myc in tamoxifeninduced apoptosis in estrogen-independent breast cancer cells. I Natl Cancer Inst 1996;88:279-84.

66 Butta A, MacLennan K, Flanders KC, et al. Induction of transforming growth factor $\beta 1$ in human breast cancer in vivo following tamoxifen treatment. Cancer Res 1992;52: 4261-4.

67 Huynh HT, Tetenes E, Wallace L, et al. In vivo inhibition of insulin-like growth factor-1 gene-expression by tamoxifen. Cancer Res 1993;53:1727-30.

68 Noguchi S, Motomura K, Inaji H, et al. Downregulation of transforming growth-factor-alpha by tamoxifen in human breast cancer. Cancer 1993;72:131-6.

69 Huynh HT, Pollak M. Insulin-like growth factor-1 gene expression in the uterus is stimulated by tamoxifen and nhibited by the pure antiestrogen ICI-182780. Cancer Res 1993;23:5585-8.

70 Huynh H, Pollak M. Uterotrophic actions of estradiol and tamoxifen are associated with inhibition of uterine insulinlike growth-factor binding protein-3 gene expression. Cancer Res 1994;54:3115-19.

71 Laatikainen TJ, Tomas EI, Voutilainen RJ. The expression of insulin-like growth-factor and its binding-protein messenger-RNA in the endometrium of postmenopausal patients with breast cancer receiving tamoxifen. Cancer 1995;76:1406-10

72 Hyder SM, Stancel GM, Chiapetta C, et al. Uterine expression of vascular endothelial growth factor is increased by estradiol and tamoxifen. Cancer Res 1996;56:3954-60.

73 Chau D, Mancoll JS, Lee S, et al. Tamoxifen downregulates TGF-beta production in keloid fibroblasts. Ann Plastic Surg 1998;40:490-3

74 Elkas J, Gray K, Howard L, et al. The effects of tamoxifen on endometrial insulin-like growth factor-1 expression. Obstet Gynecol 1998;91:45-50.

75 Zhao Y, Hague S, Manek S, et al. PCR display identifies tamoxifen induction of the novel angiogenic factor adrenomedullin by a non estrogenic mechanism in the human endometrium. Oncogene 1998;16:409-15.

76 Groom GV, Griffiths K. Effect of the anti-oestrogen tamoxifen on plasma levels of luteinizing hormone, folliclestimulating hormone, prolactin, oestradiol and progesterone in normal pre-menopausal women. $\mathcal{F}$ Endocrinol 1976; 70:421-8. 
77 Sherman BM, Chapler FK, Crickard K, et al. Endocrine consequences of continuous antiestrogen therapy with tamoxifen in premenopausal women. F Clin Invest 1979;64: 398-404

78 Patterson JS. Clinical aspects and development of antioestrogen therapy: a review of the endocrine effects of tamoxifen in animals and man. F Endocrinol 1981;89:67tamo

79 Jordan VC, Fritz NF, Tormey DC. Endocrine effects of adjuvant chemotherapy and long-term tamoxifen administration on node-positive patients with breast cancer. Cancer Res 1987;47:624-30.

80 Jordan VC, Fritz NF, Langan-Fahey S, et al. Alteration of endocrine parameters in premenopausal women with breast cancer during long-term adjuvant therapy with tamoxifen as the single agent. $\mathcal{F}$ Natl Cancer Inst 1991;83:1488-91.

81 Williams GM, Iatropoulos MJ, Djordjevic MV, et al. The triphenylethylene drug tamoxifen is a strong liver carcinogen in the rat. Carcinogenesis 1993;14:315-17.

82 Ghia $M$, Mereto E. Induction and promotion of $\gamma$-glutamyltranspeptidase-positive foci in the liver of female rats treated with ethinyl estradiol, clomiphene, tamoxifen and their associations. Cancer Lett 1989;46:195-202.
83 Yager JD, Roebuck BD, Paluscyk TL, et al. Effects of ethinyl estradiol and tamoxifen on liver DNA turnover and new synthesis and appearance of gamma glutamyl transpeptidase positive $2007-14$.

84 Dragan YP, Xu YD, Pitot HC. Tumor promotion as a target for estrogen/antiestrogen effects in rathepatic carcinogenesis. Prev Med 1991;20:15-26.

85 Newbold RR, Jefferson WN, PadillaBurgos E, et al. Uterine carcinoma in mice treated neonatally with tamoxifen. Carcinogenesis 1997;18:2293-8.

86 Han X, Liehr JG. Induction of covalent DNA adducts in rodents by tamoxifen. Cancer Res 1992;52:1360-3.

87 White INH, de Matteis F, Davies A, et al. Genotoxic potential of tamoxifen and analogues in female Fischer F344/n rats, DBA $/ 2$ and $\mathrm{C} 57 \mathrm{BL} / 6$ mice and in human $\mathrm{MCL}-5$ cells. Carcinogenesis 1992;13:2197-203.

$88 \mathrm{Li} \mathrm{D}$, Dragan Y, Jordan VC, et al. Effects of chronic administration of tamoxifen and toremifene on DNA adducts in rat liver, kidney and uterus. Cancer Res 1997;57:1438-41.

89 Carmichael PL, Ugwumadu AHN, Neven P, et al. Lack of genotoxicity of tamoxifen in human endometrium. Cancer genotoxicity of tamoxife
Res $1996 ; 56: 1475-9$.

90 Hemminki K, Rajaniemi H, Lindahl B, et al. Tamoxifen induced DNA adducts in endometrial samples from breast cancer patients. Cancer Res 1996;56:4374-7. 